\title{
Convergent subseries of divergent series
}

\author{
Marek Balcerzak ${ }^{1} \cdot$ Paolo Leonetti ${ }^{2}$ (D)
}

Received: 20 November 2020 / Accepted: 1 June 2021 / Published online: 14 June 2021

(c) The Author(s) 2021

\begin{abstract}
Let $\mathscr{X}$ be the set of positive real sequences $x=\left(x_{n}\right)$ such that the series $\sum_{n} x_{n}$ is divergent. For each $x \in \mathscr{X}$, let $\mathcal{I}_{x}$ be the collection of all $A \subseteq \mathbf{N}$ such that the subseries $\sum_{n \in A} x_{n}$ is convergent. Moreover, let $\mathscr{A}$ be the set of sequences $x \in \mathscr{X}$ such that $\lim _{n} x_{n}=0$ and $\mathcal{I}_{x} \neq \mathcal{I}_{y}$ for all sequences $y=\left(y_{n}\right) \in \mathscr{X}$ with $\liminf _{n} y_{n+1} / y_{n}>0$. We show that $\mathscr{A}$ is comeager and that contains uncountably many sequences $x$ which generate pairwise nonisomorphic ideals $\mathcal{I}_{x}$. This answers, in particular, an open question recently posed by $\mathrm{M}$. Filipczak and G. Horbaczewska.
\end{abstract}

Keywords Summable ideal · Fubini sum · Convergent series

Mathematics Subject Classification Primary: 40A05 - Secondary: 54A20

\section{Introduction}

Let $\mathscr{X}$ be the set of positive real sequences $x=\left(x_{n}\right)$ with divergent series $\sum_{n} x_{n}$. For each $x \in \mathscr{X}$, let $\mathcal{I}_{x}$ the collection of sets of positive integers $A$ such that the (possibly finite) subseries indexed by $A$ is convergent, that is,

$$
\mathcal{I}_{x}:=\left\{A \subseteq \mathbf{N}: \sum_{n \in A} x_{n}<\infty\right\} .
$$

Note that each $\mathcal{I}_{x}$ is closed under finite unions and subsets, i.e., it is an ideal. Moreover, it contains the collection Fin of finite sets $A \subseteq \mathbf{N}$, and it is different from the power set $\mathcal{P}(\mathbf{N})$. Following [4], a collection of sets of the type (1) is called summable ideal. It is not difficult

Paolo Leonetti

leonetti.paolo@gmail.com

https://sites.google.com/site/leonettipaolo/

Marek Balcerzak

marek.balcerzak@p.lodz.pl

1 Institute of Mathematics, Lodz University of Technology, ul. Wólczańska 215, 93-005 Lodz, Poland

2 Department of Decision Sciences, Università Bocconi, via Roentgen 1, 20136 Milan, Italy 
to see that every infinite set of positive integers contains an infinite subset in $\mathcal{I}_{x}$ if and only if $\lim _{n} x_{n}=0$. Accordingly, define

$$
\mathscr{Z}:=\mathscr{X} \cap c_{0}=\left\{x \in \mathscr{X}: \lim _{n \rightarrow \infty} x_{n}=0\right\} .
$$

It is known that the families $\mathcal{I}_{x}$ defined in (1) are "small", both in the measure-theoretic sense and the categorical sense, meaning that "almost all" subseries diverge, see [3, 6, $13,16]$. Related results in the context of filter convergence have been given in $[1,2,10]$. The set of limits of convergent subseries of a given series $\sum_{n} x_{n}$, which is usually called "achievement set", has been studied in [7, 9, 12]. Of special interest have been specific subseries of the harmonic series $\sum_{n} \frac{1}{n}$; see, e.g., $[11,14,15,17]$.

Roughly, the question that we are going to answer is the following: Is it true that for each $x \in \mathscr{Z}$ there exists $y \in \mathscr{X}$ such that $\mathcal{I}_{x}=\mathcal{I}_{y}$ and $\left(y_{n}\right)$ "does not oscillates too much"?

Hoping for a characterization of the class of summable ideals $\mathcal{I}_{x}$ with $x \in \mathscr{Z}$, M. Filipczak and G. Horbaczewska asked recently in [5] the following:

Question 1.1 Is it true that for each $x \in \mathscr{Z}$ there exists $y \in \mathscr{X}$ such that $\mathcal{I}_{x}=\mathcal{I}_{y}$ and

$$
\forall n \in \mathbf{N}, \quad \frac{y_{n+1}}{y_{n}} \geq \frac{n}{n+2} ?
$$

We show in Theorem 1.3 below that the answer is negative in a strong sense. To this aim, define

$$
\mathscr{Y}:=\left\{y \in \mathscr{X}: \liminf _{n \rightarrow \infty} \frac{y_{n+1}}{y_{n}}>0\right\},
$$

and note that $A-1:=\{a-1: a \in A\}$ belongs to $\mathcal{I}_{y}$ whenever $y \in \mathscr{Y}$ and $A \in \mathcal{I}_{y}$, cf. (2) below. Let also $\sim$ be the equivalence relation on $\mathscr{X}$ so that two sequences are identified if they generate the same ideal, hence

$$
\forall x, y \in \mathscr{X}, \quad x \sim y \Longleftrightarrow\left(\forall A \subseteq \mathbf{N}, \sum_{n \in A} x_{n}<\infty \Longleftrightarrow \sum_{n \in A} y_{n}<\infty\right) .
$$

First, we show that the set of pairs $(x, y) \in \mathscr{X}^{2}$ such that $x$ is $\sim$-equivalent to $y$ is topologically well behaved:

Proposition $1.2 \sim$ is a coanalytic relation on $\mathscr{X}$.

Then, we answer Question 1.1 by showing that:

Theorem 1.3 There exists $x \in \mathscr{Z}$ such that $x \nsim$ for all $y \in \mathscr{Y}$.

In light of the explicit example which will be given in the proof of Theorem 1.3, one may ask about the topological largeness of the set of such sequences. To be precise, is it true that

$$
\mathscr{A}:=\{x \in \mathscr{Z}: \forall y \in \mathscr{Y}, x \nsim y\}
$$


is a set of second Baire category, i.e., not topologically small? Note that the question is really meaningful since $\mathscr{Z}$ is completely metrizable (hence by Baire's category theorem $\mathscr{Z}$ is not meager in itself): this follows by Alexandrov's theorem [8, Theorem 3.11] and the fact that

$$
\mathscr{Z}=\bigcap_{n \geq 1}\left\{x \in c_{0}: x_{n}>0\right\} \cap \bigcap_{m \geq 1} \bigcup_{k \geq 1}\left\{x \in c_{0}: x_{1}+\cdots+x_{k}>m\right\}
$$

is a $G_{\delta}$-subset of the Polish space $c_{0}$. With the premises, we show that $\mathscr{A}$ is comeager, that is, $\mathscr{Z} \backslash \mathscr{A}$ is a set of first Baire category:

Theorem $1.4 \mathscr{A}$ is comeager in $\mathscr{Z}$. In particular, $\mathscr{A}$ is uncountable.

We remark that Theorem 1.4 gives an additional information on relation $\sim$. Since it is a coanalytic equivalence relation by Proposition 1.2, we can appeal to the deep theorem by Silver [8, Theorem 35.20] which states that every coanalytic equivalence relation on a Polish space either has countably many equivalence classes or there is a perfect set consisting of nonequivalent pairs. Thanks to Theorem 1.4, the latter holds for the relation $\sim$ in a strong form. Indeed, every pair in $\mathscr{A} \times \mathscr{Y}$ does not belong to $\sim$, where $\mathscr{A}$ is comeager (hence it contains a $G_{\delta}$-comeager subset) and $\mathscr{Y}$ is an uncountable $F_{\sigma}$-set. Therefore $\mathscr{A} \times \mathscr{Y}$ contains a product of two perfect sets by [8, Theorem 13.6].

Lastly, on a similar direction, we strenghten the fact that $\mathscr{A}$ is uncountable by proving that exist uncountably many sequences in $\mathscr{A}$ which generate pairwise nonisomorphic ideals (here, recall that two ideals $\mathcal{I}, \mathcal{J}$ are isomorphic if there exists a bijection $f: \mathbf{N} \rightarrow \mathbf{N}$ such that $f[A] \in \mathcal{I}$ if and only if $A \in \mathcal{J}$ for all $A \subseteq \mathbf{N}$ ).

Theorem 1.5 There are $\mathrm{c}$ sequences in $\mathscr{A}$ which generate pairwise nonisomorphic ideals.

Hereafter, we use the convention that $\sum_{n \geq 1} a_{n} \ll \sum_{n \geq 1} b_{n}$, with each $a_{n}, b_{n}>0$, is a shorthand for the existence of $C>0$ such that $\sum_{n \leq k} a_{n} \leq C \sum_{n \leq k} b_{n}$ for all $k \in \mathbf{N}$.

\section{Proof of Proposition 1.2}

Equivalently, we have to show that the set $E:=\left\{(x, y) \in \mathscr{X}^{2}: x \nsim y\right\}$ is analytic in $\mathscr{X}^{2}$. For, note that $E$ is the projection on $\mathscr{X}^{2}$ of $E_{1} \cup E_{2}$, where

$$
E_{1}:=\left\{(A, x, y) \in \mathcal{P}(\mathbf{N}) \times \mathscr{X}^{2}: A \in \mathcal{I}_{x} \backslash \mathcal{I}_{y}\right\}
$$

and, similarly,

$$
E_{2}:=\left\{(A, x, y) \in \mathcal{P}(\mathbf{N}) \times \mathscr{X}^{2}: A \in \mathcal{I}_{y} \backslash \mathcal{I}_{x}\right\}
$$

Now, for each $n \in \mathbf{N}$, define the functions $\alpha_{n}, \beta_{n}: \mathcal{P}(\mathbf{N}) \times \mathscr{X}^{2} \rightarrow \mathbf{R}$ by $\alpha_{n}(A, x, y)=\sum x_{t}$ and $\beta_{n}(A, x, y)=\sum y_{t}$, where each sum is extended over all $t \in A$ such that $t \leq n$. Since they are continuous, the set $\left(\alpha_{n} \leq k\right):=\left\{(A, x, y) \in \mathcal{P}(\mathbf{N}) \times \mathscr{X}^{2}: \alpha_{n}(A, x, y) \leq k\right\}$ is closed and $\left(\beta_{n}>k\right)$ is open for all $n, k \in \mathbf{N}$. Therefore 


$$
E_{1}=\left(\bigcup_{k \geq 1} \bigcap_{n \geq 1}\left(\alpha_{n} \leq k\right)\right) \cap\left(\bigcap_{k \geq 1}\left(\beta_{n \geq 1}>k\right)\right)
$$

is the intersection of an $F_{\sigma}$-set and a $G_{\delta}$-set, hence it is Borel. Analogously, $E_{2}$ is Borel. This proves that $E$ is analytic subset of $\mathscr{X}^{2}$.

\section{Proof of Theorem 1.3}

Define the sequence $x=\left(x_{n}\right)$ so that $x_{n}=\frac{1}{n}$ if $n$ is even and $x_{n}=\frac{1}{n \log (n+1)}$ if $n$ is odd. Note that $\lim _{n} x_{n}=0$ and that $\sum_{n} x_{n}=\infty$, hence $x \in \mathscr{Z}$. At this point, fix $y \in \mathscr{Y}$ such that $\kappa:=\liminf _{n} y_{n+1} / y_{n}>0$ and let us show that $\mathcal{I}_{x} \neq \mathcal{I}_{y}$.

Let $\mathbf{P}$ be the set of prime numbers, with increasing enumeration $\left(p_{n}\right)$. By the prime number theorem we have $p_{n}$ is asymptotically equal to $n \log (n)$ as $n \rightarrow \infty$, hence

$$
\sum_{n \in \mathbf{P}} x_{n}=\sum_{n \geq 1} x_{p_{n}} \ll \sum_{n \geq 1} \frac{1}{p_{n} \log \left(p_{n}\right)} \ll \sum_{n \geq 2} \frac{1}{n \log ^{2}(n)}<\infty,
$$

with the consequence that $\mathbf{P} \in \mathcal{I}_{x}$. In addition, $\mathbf{P}-1 \notin \mathcal{I}_{x}$ because

$$
\sum_{n \in \mathbf{P}-1} x_{n}=\sum_{n \geq 1} x_{p_{n}-1} \gg \sum_{n \geq 1} \frac{1}{p_{n}} \gg \sum_{n \geq 2} \frac{1}{n \log (n)}=\infty .
$$

Lastly, suppose for the sake of contradiction that $\mathcal{I}_{x}=\mathcal{I}_{y}$. Then we should have that $\mathbf{P} \in \mathcal{I}_{y}$ and, at the same time, $\mathbf{P}-1 \notin \mathcal{I}_{y}$. The latter means that

$$
\sum_{n \geq 1} y_{p_{n}-1}=\infty
$$

However, this implies that

$$
\sum_{n \geq 1} y_{p_{n}} \gg \sum_{n \geq 1} \kappa y_{p_{n}-1}=\infty
$$

contradicting that $\mathbf{P} \in \mathcal{I}_{y}$.

\section{Proof of Theorem 1.4}

Consider the Banach-Mazur game defined as follows: Players I and II choose alternatively nonempty open subsets of $\mathscr{Z}$ as a nonincreasing chain $U_{1} \supseteq V_{1} \supseteq U_{2} \supseteq V_{2} \supseteq \cdots$, where Player I chooses the sets $U_{m}$. Player II has a winning strategy if $\bigcap_{m} V_{m} \subseteq \mathscr{A}$. Thanks to [8, Theorem 8.33], Player II has a winning strategy if and only if $\mathscr{A}$ is comeager. Hence, the rest of the proof consists in showing that Player II has a winning strategy.

Note that the open neighborhood of a sequence $x \in \mathscr{Z}$ with radius $\varepsilon>0$ satisfies

$$
B_{\varepsilon}(x):=\{y \in \mathscr{Z}:\|x-y\|<\varepsilon\} \supseteq\left\{y \in \mathscr{Z}: \forall n \in \mathbf{N},\left|x_{n}-y_{n}\right|<\frac{\varepsilon}{2}\right\} .
$$

Since $x \in \mathscr{Z} \subseteq c_{0}$, there exists $k_{0}=k_{0}(x, \varepsilon) \in \mathbf{N}$ such that $x_{n}<\frac{\varepsilon}{2}$ for all $n \geq k_{0}$. Hence 


$$
B_{\varepsilon}(x) \supseteq W_{\varepsilon}(x):=\left\{y \in \mathscr{Z}: \forall n \geq k_{0}(x, \varepsilon), y_{n}<\frac{\varepsilon}{2} \text { and } \forall n<k_{0}(x, \varepsilon),\left|x_{n}-y_{n}\right|<\frac{\varepsilon}{2}\right\} .
$$

For each $m \in \mathbf{N}$, suppose that the nonempty open set $U_{m}$ has been fixed by Player I. Hence, $U_{m}$ contains an open ball $B_{\varepsilon_{m}}\left(x^{(m)}\right)$, for some $x^{(m)} \in \mathscr{Z}$ and $\varepsilon_{m}>0$. In particular, thanks to (3), there exists a sufficiently large integer $k_{0}=k_{0}\left(x^{(m)}, \varepsilon_{m}\right) \in \mathbf{N}$ such that $y_{n}<\frac{\varepsilon_{m}}{2}$ for all $y \in W_{\varepsilon_{m}}\left(x^{(m)}\right)$ and $n \geq k_{0}$. Without loss of generality, let us suppose that $k_{0}$ is even.

At this point, let $x^{\star}$ be the sequence in $\mathscr{A}$ defined in the proof of Theorem 1.3. Then, for each $m \in \mathbf{N}$, let $t_{m} \geq k_{0}\left(x^{(m)}, \frac{\varepsilon_{m}}{2}\right.$ ) be an integer such that $\max \left\{x_{p_{m}}^{\star}, x_{p_{m}-1}^{\star}\right\}<t_{m} \cdot \frac{\varepsilon_{m}}{4}$ (we recall that $p_{m}$ stands for the $m$ th prime number), and define the positive real

$$
\delta_{m}:=\min \left\{\frac{1}{m^{2} t_{m}}, \frac{\varepsilon_{m}}{4}-\frac{\max \left\{x_{p_{m}}^{\star}, x_{p_{m}-1}^{\star}\right\}}{t_{m}}\right\} .
$$

Note that $\lim _{m} \delta_{m}=0$. Without loss of generality, we can assume also that $k_{0}\left(x^{(m+1)}, \varepsilon_{m+1}\right)>k_{0}\left(x^{(m)}, \varepsilon_{m}\right)+2 t_{m}$ for all $m \in \mathbf{N}$. Now, define the set $I_{m}=\mathbf{N} \cap\left[k_{0}\left(x^{(m)}, \varepsilon_{m}\right), k_{0}\left(x^{(m)}, \varepsilon_{m}\right)+2 t_{m}\right)$ (hence the sets $I_{m}$ are pairwise disjoint) and let $z^{(m)}$ be the sequence such that

$$
\forall n \in \mathbf{N} \quad z_{n}^{(m)}= \begin{cases}x_{p_{m}-1}^{\star} / t_{m} & \text { if } n \in I_{m} \text { and } n \text { even, } \\ x_{p_{m}}^{\star} / t_{m} & \text { if } n \in I_{m} \text { and } n \text { odd, } \\ x_{n}^{(m)} & \text { if } n \notin I_{m} .\end{cases}
$$

Lastly, set $V_{m}:=B_{\delta_{m}}\left(z^{(m)}\right)$ and note that, for each sequence $y \in V_{m}$, we have by construction:

(i) $\left|y_{n}-x_{n}^{(m)}\right|=\left|y_{n}-z_{n}^{(m)}\right|<\delta_{m}<\frac{\varepsilon_{m}}{2}$ if $n<\min I_{m}$;

(ii) $y_{n}<z_{n}^{(m)}+\delta_{m} \leq \max \left\{x_{p_{m}-1}^{\star}, x_{p_{m}}^{\star}\right\} / t_{m}+\delta_{m}<\frac{\varepsilon_{m}}{2}$ if $n \in I_{m}$;

(iii) $y_{n}<z_{n}^{(m)}+\delta_{m}=x_{n}^{(m)}+\delta_{m}<\frac{\varepsilon_{m}}{4}+\frac{\varepsilon_{m}}{4}=\frac{\varepsilon_{m}}{2}$ if $n>\max I_{m}$.

(In the last point, we used that if $n>\max I_{m} \geq t_{m} \geq k_{0}\left(x^{(m)}, \frac{\varepsilon_{m}}{2}\right)$ then $x_{n}^{(m)} \leq \frac{1}{2} \cdot \frac{\varepsilon_{m}}{2}$.) This implies that $y \in W_{\varepsilon_{m}}\left(x^{(m)}\right)$. To sum up, we obtain

$$
\forall m \in \mathbf{N}, \quad V_{m} \subseteq W_{\varepsilon_{m}}\left(x^{(m)}\right) \subseteq B_{\varepsilon_{m}}\left(x^{(m)}\right) \subseteq U_{m},
$$

hence $V_{m}$ is a nonempty open set contained in $U_{m}$. In addition, the sequence of centers $\left(z^{(m)}\right)$ is a Cauchy sequence in the complete metric space $\mathscr{Z}$. Indeed, since the sequence $\left(V_{n}\right)$ is decreasing, we have $\left\|z^{(m)}-z^{\left(m^{\prime}\right)}\right\| \leq \delta_{m} \leq \frac{1}{m^{2}}$ for all integers $m^{\prime} \geq m \geq 1$. Hence $\left(z^{(m)}\right)$ is convergent to some $z \in \mathscr{Z}$ and it is straighforward to see that $\{z\}=\bigcap_{m} V_{m}$.

To complete the proof, we need to show that $z \in \mathscr{A}$. Set $A:=\left(\bigcup_{m} I_{m}\right) \backslash 2 N$. Proceeding as in the proof of Theorem 1.3, we see that

$$
\sum_{n \in A} z_{n}=\sum_{m \geq 1} \sum_{n \in I_{m} \backslash 2 \mathbf{N}} z_{n} \leq \sum_{m \geq 1} \sum_{n \in I_{m} \backslash 2 \mathbf{N}}\left(z_{n}^{(m)}+\delta_{m}\right) \leq \sum_{m \geq 1}\left|I_{m}\right|\left(\frac{x_{p_{m}}^{\star}}{t_{m}}+\frac{1}{m^{2} t_{m}}\right)<\infty,
$$

hence $A \in \mathcal{I}_{z}$. Similarly, $A-1 \notin \mathcal{I}_{z}$ since 


$$
\sum_{n \in A-1} z_{n} \geq \sum_{m \geq 1} \sum_{n \in I_{m} \cap 2 \mathbf{N}}\left(z_{n}^{(m)}-\delta_{m}\right) \gg \sum_{m \geq 1}\left|I_{m}\right|\left(\frac{x_{p_{m}}^{\star}-1}{t_{m}}-\frac{1}{m^{2} t_{m}}\right)=\infty .
$$

Now, fix $y \in \mathscr{Y}$ such that $\kappa:=\liminf _{n} y_{n+1} / y_{n}>0$ and suppose that $\mathcal{I}_{z}=\mathcal{I}_{y}$. Then we would have that $A \in \mathcal{I}_{y}$ and $A-1 \notin \mathcal{I}_{y}$, which is impossible reasoning as in (2).

\section{Proof of Theorem 1.5}

Let $x^{\star}$ be the sequence defined in the proof of Theorem 1.3. For each $r \in(0,1]$, let $x^{(r)}$ be the sequence defined by $x_{n}^{(r)}=\left(x_{n}^{\star}\right)^{r}$ for all $n \in \mathbf{N}$. Replacing the set of primes $\mathbf{P}$ with

$$
\mathbf{P}_{r}:=\left\{2\left\lfloor\frac{p_{n}^{1 / r}}{2}\right\rfloor+1: n \in \mathbf{N}\right\}
$$

we obtain that

$$
\sum_{n \in \mathbf{P}_{r}} x_{n}^{(r)} \ll \sum_{n \geq 1}\left(\frac{1}{p_{n}^{1 / r} \log \left(p_{n}^{1 / r}\right)}\right)^{r} \ll \sum_{n \geq 1} \frac{1}{n(\log (n))^{1+r}}<\infty,
$$

and $\sum_{n \in \mathbf{P}_{r}-1} x_{n}^{(r)} \gg \sum_{n \geq 1} \frac{1}{p_{n}}=\infty$. Reasoning as in the proof of Theorem 1.3, we conclude that $x^{(r)} \in \mathscr{A}$ for each $r \in(0,1]$.

To complete the proof, fix reals $r, s$ such that $0<r<s \leq 1$. Then, it is sufficient to show that the ideals generated by $x^{(r)}$ and $x^{(s)}$ are not isomorphic. To this aim, let $f: \mathbf{N} \rightarrow \mathbf{N}$ be a bijection and assume for the sake contradiction that

$$
\forall A \subseteq \mathbf{N}, \quad \sum_{n \in A} x_{f(n)}^{(r)}<\infty \text { if and only if } \sum_{n \in A} x_{n}^{(s)}<\infty .
$$

Fix $t \in\left(1, \frac{s}{r}\right)$ and define $S:=\left\{n \in \mathbf{N}: f(n)>n^{t}\right\}$ and $T:=\mathbf{N} \backslash S$. We have $\sum_{n \in S} \frac{1}{f(n)} \leq \sum_{n \in S} \frac{1}{n^{t}}<\infty$. Considering that $f$ is a bijection and the harmonic series is divergent, we obtain that $\sum_{n \in T} \frac{1}{f(n)}=\infty$ (in particular, $T$ is infinite). In addition, since $r<1$ and

$$
\frac{1}{f(n)} \ll \frac{1}{(f(n) \log (f(n)+1))^{r}} \leq x_{f(n)}^{(r)} \leq \frac{1}{f^{r}(n)},
$$

we get that $\sum_{n \in T} x_{f(n)}^{(r)}=\infty$ and, thanks to (4), also that $\sum_{n \in T} x_{n}^{(s)}=\infty$. Note that, if $n \in T$, then $f(n) \leq n^{t}$, which implies that

$$
\forall n \in T, \quad \frac{x_{n}^{(s)}}{x_{f(n)}^{(r)}} \leq \frac{1 / n^{s}}{1 /(f(n) \log (f(n)+1))^{r}} \ll \frac{(\log n)^{r}}{n^{s-t r}},
$$

which has limit 0 if $n \rightarrow \infty$ (and belongs to $T$ ). In particular, for each $k \in \mathbf{N}$, there exists $n_{k} \in \mathbf{N}$ such that $x_{n}^{(s)} / x_{f(n)}^{(r)} \leq \frac{1}{k^{2}}$ for all $n \geq n_{k}$. Let $\left(A_{k}\right)$ be a sequence of finite subsets of $T$ defined recursively as follows: for each $k \in \mathbf{N}$, let $A_{k}$ be a finite subset of $T$ such that $\min A_{k} \geq n_{k}+\max A_{k-1}$ and $\sum_{n \in A_{k}} x_{f(n)}^{(r)} \in\left(\frac{1}{2}, 1\right)$ where, by convention, we assume $\max A_{0}:=0$ (note that it is really possible to define such sequence). Finally, define $A:=\bigcup_{k} A_{k}$ so that we obtain 


$$
\sum_{n \in A} x_{f(n)}^{(r)}=\sum_{k \geq 1} \sum_{n \in A_{k}} x_{f(n)}^{(r)}=\infty \text { and } \sum_{n \in A} x_{n}^{(s)} \leq \sum_{k \geq 1} \sum_{n \in A_{k}} \frac{x_{f(n)}^{(r)}}{k^{2}} \leq \sum_{k \geq 1} \frac{1}{k^{2}}<\infty .
$$

This contradicts (4), concluding the proof.

\section{Concluding remarks}

We remark that the ideal $\mathcal{I}_{x}$ defined in the proof above is just (an isomorphic copy of) the Fubini sum $\mathcal{I}_{s} \oplus \mathcal{I}_{t}$, where $s, t \in \mathscr{Z}$ are sequences defined by $s_{n}=\frac{1}{n}$ and $t_{n}=\frac{1}{n \log (n+1)}$ for all $n \in \mathbf{N}$. Here, we recall that the Fubini sum of two ideals $\mathcal{I}$ and $\mathcal{J}$ on $\mathbf{N}$ is the ideal $\mathcal{I} \oplus \mathcal{J}$ on $\{0,1\} \times \mathbf{N}$ of all sets $A$ such that $\{n \in \mathbf{N}:(0, n) \in A\} \in \mathcal{I}$ and $\{n \in \mathbf{N}:(1, n) \in A\} \in \mathcal{J}$, cf. e.g. [4, p. 8]

Also, some comments are in order about the simplifications. The assumption that the sequence $x$ has positive elements (instead of nonnegative elements) is rather innocuous. Indeed, in the opposite, if $x_{n}=0$ for infinitely many $n$, then the summable ideal $\mathcal{I}_{x}$ would be (isomorphic to) the Fubini sum $\mathcal{P}(\mathbf{N}) \oplus \mathcal{I}_{y}$, for some $y \in \mathscr{X}$. Lastly, also the hypothesis $x \in \mathscr{Z}$ in Question 1.1 (instead of $x \in \mathscr{X}$ ) has a similar justification. Indeed, if $x \in \mathscr{X} \backslash \mathscr{Z}$, then $\mathcal{I}_{x}$ would be the Fubini sum Fin $\oplus \mathcal{I}_{y}$, for some $y \in \mathscr{Z}$.

We conclude with two open questions:

Question 6.1 Is it true that for each $x \in \mathscr{Z}$ there are (possibly infinite) sequences $y^{1}, y^{2}, \ldots \in \mathscr{Y}$ such that $\mathcal{I}_{x}=\bigoplus_{i} \mathcal{I}_{y^{i}}$ ?

Question 6.2 Is it true that $\mathscr{A}$ is an analytic subset of $\mathscr{Z}$ ?

Acknowledgements The authors are grateful to two anonymous referees for several constructive comments which improved the exposition of the article.

Funding Open access funding provided by Università Commerciale Luigi Bocconi within the CRUI-CARE Agreement. P.L. is grateful to PRIN 2017 (grant 2017CY2NCA) for financial support.

Open Access This article is licensed under a Creative Commons Attribution 4.0 International License, which permits use, sharing, adaptation, distribution and reproduction in any medium or format, as long as you give appropriate credit to the original author(s) and the source, provide a link to the Creative Commons licence, and indicate if changes were made. The images or other third party material in this article are included in the article's Creative Commons licence, unless indicated otherwise in a credit line to the material. If material is not included in the article's Creative Commons licence and your intended use is not permitted by statutory regulation or exceeds the permitted use, you will need to obtain permission directly from the copyright holder. To view a copy of this licence, visit http://creativecommons.org/licenses/by/4.0/.

\section{References}

1. Balcerzak, M., Popławski, M., Wachowicz, A.: The Baire category of ideal convergent subseries and rearrangements. Topol. Appl. 231, 219-230 (2017)

2. Balcerzak, M., Popławski, M., Wachowicz, A.: Ideal convergent subseries in Banach spaces. Quaest. Math. 42(6), 765-779 (2019)

3. Buck, R.C.: Limit points of subsequences. Bull. Amer. Math. Soc. 50, 395-397 (1944) 
4. Farah, I.: Analytic quotients: theory of liftings for quotients over analytic ideals on the integers. Mem. Amer. Math. Soc. 148(702), xvi+177 (2000)

5. Filipczak, M., Horbaczewska, G.: Some kinds of sparseness on the real line and ideals on $\omega$. Ann. Math. Sil. 34(1), 45-50 (2020)

6. Ganguly, D.K., Dutta, C.: Some remarks on subseries of divergent series. Soochow J. Math. 31(4), 537-542 (2005)

7. Jones, R.: Achievement sets of sequences. Am. Math. Monthly 118(6), 508-521 (2011)

8. Kechris, A.S.: Classical Descriptive Set Theory, Graduate Texts in Mathematics, vol. 156. SpringerVerlag, New York (1995)

9. Leonetti, P.: Convergence rates of subseries. Am. Math. Monthly 126(2), 163-167 (2019)

10. Leonetti, P., Miller, H., Miller-Van Wieren, L.: Duality between measure and category of almost all subsequences of a given sequence. Period. Math. Hungar. 78(2), 152-156 (2019)

11. Lubeck, B., Ponomarenko, V.: Subsums of the harmonic series. Am. Math. Monthly 125(4), 351-355 (2018)

12. Nitecki, Z.: Cantorvals and subsum sets of null sequences. Am. Math. Monthly 122(9), 862-870 (2015)

13. Rao, M.B., Rao, K.P.S.B., Rao, B.V.: Remarks on subsequences, subseries and rearrangements. Proc. Am. Math. Soc. 67(2), 293-296 (1977)

14. Schmelzer, T., Baillie, R.: Summing a curious, slowly convergent series. Am. Math. Monthly 115(6), 525-540 (2008)

15. Stadje, W.: On the convergence of thinned harmonic series. Elem. Math. 70(3), 89-94 (2015)

16. Šalát, T.: On subseries. Math. Z. 85, 209-225 (1964)

17. Wadhwa, A.D.: Some convergent subseries of the harmonic series. Am. Math. Monthly 85(8), 661663 (1978)

Publisher's Note Springer Nature remains neutral with regard to jurisdictional claims in published maps and institutional affiliations. 